\title{
Flexibilidad laboral en el trabajo investigativo: auxiliares de investigación
}

\author{
Elizabeth Boscán* \\ Lilia Pereira de Homes**
}

\section{Resumen}

Esta investigación tiene como objetivo explorar las condiciones de trabajo de los profesionales que laboran en calidad de auxiliares de investigación en los proyectos o programas adscritos a las unidades, centros e institutos de investigación de la Universidad del Zulia, Venezuela, financiados por el Consejo de Desarrollo Cientifico y Humanístico. La metodología de carácter exploratoria-descriptiva se basó en la revisión de protocolos de proyectos y programas terminados en los años 2002 y 2003; asimismo, se realizaron entrevistas a una muestra de auxiliares de investigación y a profesores responsables de proyectos. Los resultados revelan que las condiciones laborales de este tipo de personal presentan bajos ingresos, inseguridad en la continuidad laboral y ausencia de protección social. Se concluye que el trabajo para el personal de apoyo tiene escasos incentivos económicos, mientras que el estímulo esencial lo constituyen las posibilidades de formación teórica-metodológica necesaria para el desarrollo de las aptitudes investigativas.

Palabras clave: precariedad laboral, trabajo investigativo, auxiliares de investigación, Consejo de Desarrollo Científico y Humanístico, Universidad del Zulia.

\section{Introducción}

$\mathrm{L}$ a investigación es una actividad estrechamente relacionada con las universidades; en las de Venezuela se produce $70 \%$ de los conocimientos; sin embargo, en el nivel mundial existe una marcada tendencia al desplazamiento de tales instituciones como únicos centros de acumulación de saberes.

* Estudiante de la maestría en Gerencia Pública de la Facultad de Ciencias Económicas y Sociales de la Universidad del Zulia, Venezuela. Correo electrónico: elizabeth_boscan@yahoo.es

** Profesora investigadora de la Facultad de Ciencias Económicas y Sociales de la Universidad del Zulia, Venezuela. Correo electrónico: 1pereira14@yahoo.es 
El proceso de creación de conocimientos es una actividad inherente al trabajo del personal docente y de investigación, que se lleva a cabo en unidades, centros e institutos de investigación. La función de investigación se realiza a través de proyectos o programas financiados por diferentes organismos que tienen como función primordial promover la investigación en el ámbito nacional, básicamente a través del Fondo Nacional de Ciencia y Tecnología (FONACIT) y en el ámbito universitario por los Consejos de Desarrollo Científico y Humanístico $\left(\mathrm{CONDES}^{1}\right)$.

La unidad fundamental de trabajo en los programas y proyectos ${ }^{2}$ son los equipos de investigación integrados fundamentalmente por un investigador responsable, un coinvestigador principal, coinvestigadores auxiliares y personal de apoyo a la investigación. Dentro de estos últimos se encuentran los auxiliares de investigación, egresados universitarios, quienes deben cumplir diversas actividades inherentes al proceso investigativo tales como recolección y procesamiento de información, realización de entrevistas, informes administrativos exigidos por el CONDES, participación en discusiones teóricas y metodológicas inherentes al objeto de estudio. Este recurso humano representa una fuerza laboral subordinada al investigador responsable, quien administra los fondos asignados a la investigación y gestiona "la remuneración" que perciben.

A partir de lo anterior y considerando que la investigación es una actividad marginada en el ámbito universitario, el presente trabajo describe las condiciones bajo las cuales laboran los auxiliares de investigación, atendiendo a características propias de un mercado laboral cada vez más flexible con tendencia a la precarización del trabajo.

La metodología utilizada fue la exploratoria-descriptiva con base en la revisión de protocolos de proyectos y programas finalizados en 2002 y 2003; además, se realizaron entrevistas a una muestra de auxiliares de investigación y a profesores responsables de proyectos.

1 Esta instancia de promoción y coordinación de la investigación en otras universidades nacionales se conoce como CDCH o CDCHT.

${ }^{2}$ Un programa de investigación es la reunión o conjunto de tres a cinco proyectos simultáneos y relacionados entre sí en función de un área determinada del conocimiento. Por su parte, un proyecto representa la conformación de un equipo de trabajo para trabajar en alguna temática del conocimiento. 
Flexibilidad laboral en el trabajo

investigativo: auxiliares de investigación

\section{Aspectos preliminares}

La precariedad laboral es un fenómeno conexo con la flexibilización laboral; es definida por algunos autores como una relación causa-efecto, estrechamente relacionada con la adopción de medidas neoliberales impulsadas por el fenómeno globalizador. Tales medidas ubican al mercado como regulador de las relaciones laborales en contraposición al modelo de estado de bienestar vigente en América Latina desde los años cincuenta; en dicho modelo el Estado fungió como protector de los derechos laborales para garantizar la reproducción de la fuerza de trabajo.

En Venezuela durante el estado de bienestar la administración pública se organizó con base en una política de expansión a través de la creación de empresas públicas y la creación de instituciones sociales especialmente en el campo de la salud y la educación (Córdova, 2000:132).

Los ingentes ingresos por renta petrolera en los setenta ${ }^{3}$ promovieron la capacidad del Estado para satisfacer demandas sociales y de inversión; en esa década, los años entre 1974 y 1977 son considerados por muchos autores como la etapa del boom petrolero. ${ }^{4}$ En el ámbito laboral se promovió la contratación colectiva mediante una política de pactos entre empresarios y trabajadores. De acuerdo con Jaua (1997:89), se buscaba garantizar estabilidad, salario pactado, jornada laboral y condiciones idóneas de seguridad e higiene industrial en la contratación colectiva, siendo esta última la condición distintiva de la lógica del estado de bienestar.

Posteriormente, en las décadas de los ochenta y noventa, el discurso de carácter neoliberal adquiere fuerza en América Latina debido al agotamiento del modelo sustitutivo de importaciones. El Estado se colapsó como agente distribuidor de la renta petrolera, situación que se refleja en el deterioro progresivo de la economía.

En Venezuela, las iniciativas neoliberales tienen su eco a inicios de la década de los ochenta y se acentúan a finales de la misma. La adopción del paquete de ajustes macroeconómicos propugnaba la desregulación laboral, reducción del Estado, aper-

\footnotetext{
${ }^{3}$ En Venezuela el ingreso nacional alcanzó en 1970 la cifra de 39,894 millones de bolívares; en 1972, 50,152; en 1974, 101,512; en 1977, 140,932; y en 1978, 153,852 (Banco Central de Venezuela).

${ }^{4}$ El barril de petróleo en 1973 se vendió a \$2,77 y en 1974 a \$ 9,18 lo cual representó un aumento de 231, 4\% (Banco Central de Venezuela).
} 
tura a los mercados internacionales, entre otras. Estas medidas de ajuste estructural se aplicaron parcialmente, siendo la caja de resonancia la promoción de la apertura petrolera. En otros sectores, como salud y educación, se anunciaron medidas privatizadoras, pero buena parte de ellas quedaron en el ámbito propositivo.

La doctrina neoliberal ubica al mercado como ente regulador de las relaciones laborales. Ello genera, según Suárez et al. (2001:383), modificaciones en las relaciones de trabajo expresadas en nuevas políticas de contratación de personal, modificación en el contenido de las calificaciones requeridas, ajustes en el número de trabajadores de la empresa de acuerdo con los cambios de la demanda, reducción del tiempo de trabajo por la adopción de nuevas tecnologías.

En este contexto globalizador, la eficiencia es el criterio central que rige la actividad productiva, siendo la flexibilización laboral una de las estrategias utilizadas en la relación capital-trabajo. En el sector público, la flexibilidad se presenta como una opción para superar la rigidez de las formas de contratación del trabajo típicas de este sector. No obstante, las universidades nacionales representan un sector laboral en el cual todavía se conservan las relaciones laborales de carácter tradicional típicas del estado de bienestar. Señala Ermida (1999:137) que este tipo de relaciones laborales se caracteriza por la relación empleado-trabajador por tiempo indeterminado; ingreso con vocación de continuidad y existencia del sindicato para la regulación de los conflictos laborales, cuyo fin es proteger a la parte afectada - el trabajador.

El hecho de que prevalezca el tipo de relación descrito anteriormente ha significado una pesada carga burocrática ${ }^{5}$ en el mantenimiento del personal que labora en las universidades nacionales. Esta rigidez laboral ha sido reforzada por un modelo de gestión universitario que Ferrer (2000:387) caracteriza como clientelar, con estricto apego a la jerarquía y autorreferenciado, aspectos que conllevan a un laissez-faire en la actuación universitaria.

En este modelo, la función investigativa de los profesores se ha visto afectada por el privilegio que ha tenido la función docente como respuesta al incremento sostenido de estudiantes a la educación superior. En esta situación han contribuido

${ }_{5}$ El 80\% del presupuesto universitario de las universidades oficiales se destina al pago de la nómina del personal docente, administrativo y obrero (Lombardi, 2004). 
Flexibilidad laboral en el trabajo

investigativo: auxiliares de investigación

también factores de carácter social tales como la escasa apreciación social que tiene la investigación como trabajo y el científico como trabajador. Al respecto señala Gaillard (1994:254) que la investigación no se considera todavía una profesión del todo aceptable. Los científicos no gozan de un nivel social o de prestigio alto comparado con otros profesionales del mismo nivel académico (médicos, abogados, ingenieros).

En consecuencia, la conformación de una sólida comunidad científica y el arraigo de la profesión académica con base en la investigación han dificultado su institucionalización, a lo cual se adicionan otros factores tales como las débiles condiciones en cuanto a instalaciones, tecnología, políticas de incentivo al rendimiento, apoyo bibliográfico y actualización académica.

Sin embargo, se destaca una gradual conformación de comunidades científicas académicas que logran superar adversidades y producir conocimientos científico, tecnológico y humanístico. En la mayoría de estas comunidades se incorpora personal de apoyo a la investigación.

Ahora bien, en la Universidad del Zulia se desarrollan proyectos y programas de investigación adscritos a centros e institutos de investigación. En los financiados por el CONDES, los egresados universitarios tienen la oportunidad de incorporarse al trabajo investigativo como auxiliares de investigación. Estos profesionales se encuentran en condiciones de trabajo desventajosas con tendencia a la flexibilidad laboral.

En la siguiente sección discutiremos acerca de las funciones inherentes al papel que desempeña el auxiliar de investigación en los diversos programas y proyectos financiados por el CONDES.

\section{El rol del auxiliar de investigación}

El auxiliar de investigación "[...] es un personal de apoyo a la investigación que presta servicios profesionales para el desarrollo de actividades específicas, con cargo al Programa/Proyecto"(CONDES, 2004). Su proceso de captación se realiza por diversas vías, algunos investigadores lo hacen entre los estudiantes de pregrado que realizan el trabajo especial de grado o pasantías en los programas o 
proyectos adscritos a unidades, centros e institutos de investigación de la universidad; o también entre los estudiantes próximos a grado académico que tengan inquietudes indagatorias y manifiesten su interés por incursionar en la actividad investigativa. De ambas maneras, este personal ingresa en condición de colaborador como estudiantes de pregrado o como auxiliares de investigación una vez egresados del pregrado.

Las actividades del auxiliar de investigación, al igual que la del resto del personal de apoyo, no están explícitas en los documentos normativos del CONDES. Sin embargo, de acuerdo con la información suministrada por los propios auxiliares de investigación y por la revisión de protocolos de proyectos y programas que se efectuó, se evidenció que entre las actividades que le son asignadas se encuentran las siguientes: recolección de información, trabajo de campo, realización de entrevistas, gestión de trámites administrativos, presentación de ponencias en eventos nacionales y otras actividades requeridas en el desarrollo del proyecto. A pesar de que la mayor proporción de las actividades que realizan son de carácter operativo, su participación en actividades de valor científico queda a discreción de los investigadores; sin embargo, podemos señalar las siguientes: participación en las discusiones teóricas y metodológicas, que se llevan a cabo en el equipo de investigación; realización de aportes teóricos-empíricos, que podrían conllevar a la coautoría de ponencias y artículos científicos; participación en talleres dirigidos a la formación en investigación y asistencia como ponentes a eventos científicos nacionales.

Por su parte, los investigadores responsables, aun cuando no se detalla en la normativa universitaria, tienen entre sus funciones la responsabilidad de la formación teórica metodológica de los integrantes del grupo de investigación y del personal de apoyo; además, dirigir y supervisar las actividades inherentes al proceso investigativo del proyecto o programa.

La inexistencia de descripción de tareas y funciones para este personal genera desigualdades en el tipo de actividades asignadas; por ello, se encontró en la revisión que se realizó del informe final de los proyectos y programas financiados por el CONDES y finalizados en los años 2003 y 2004 que sólo un 33\% de ese personal figuraba como coautor en ponencias y un $60 \%$ en publicaciones; es decir que 
Flexibilidad laboral en el trabajo

investigativo: auxiliares de investigación

mientras unos participaron activamente en la actividad científica otros no lo hicieron. Los primeros son los que mayormente tienen la oportunidad de formarse en investigación e ir conformando un perfil cónsono con la actividad científica.

Si se toma en consideración el hecho de que los auxiliares de investigación representan una posible fuente de generación de relevo, se hace imprescindible proporcionarle formación teórica-metodológica durante su permanencia en el proyecto/ programa para su participación en las actividades creativas como son la coautoría en ponencias o artículos. De lo contrario, se le coarta la posibilidad de recibir apoyo financiero por parte del CONDES para proseguir su formación académica en los posgrados que ofrece LUZ, pues aquella instancia exige como requisito para financiamiento poseer al menos un artículo publicado y tener dos años de experiencia en investigación.

Con respecto al financiamiento para que los auxiliares de investigación asistan a eventos científicos nacionales y cursen estudios de posgrado, se exigen los mismos requisitos señalados anteriormente. La condición sine qua non de ser investigador activo para el disfrute de los beneficios que plantea el CONDES nos lleva a reflexionar respecto al marco normativo vigente en LUZ, el cual induce a los investigadores a proporcionar formación en investigación al personal de apoyo. Se deduce entonces que el objetivo final es la contribución a la conformación de una joven comunidad científica, estrategia que favorecería el fortalecimiento de la función investigativa.

Ahora bien, existen circunstancias que confinan al auxiliar de investigación hacia un rol instrumental, dada la discrecionalidad del investigador responsable en la asignación de actividades y también por la inexistencia de descripción de funciones y la ausencia de una política de formación emanada desde el CONDES para el personal de apoyo. El resultado se traduce en un desperdicio de recursos y esfuerzos para ambos (investigadores y auxiliares).

La vulnerabilidad profesional que afecta a los auxiliares de investigación es consecuencia de la escasa institucionalización de la ciencia en Venezuela especialmente en las universidades. En el caso de la Universidad del Zulia es una actividad "marginada" a la cual se otorgan incipientes recursos económicos; además, se encuentra subordinada a la actividad docente. Sólo unos pocos se sienten atraídos por estos cargos. 
El atractivo económico que ofrece el trabajo de auxiliar de investigación dentro del mercado laboral académico es escaso; sin embargo —según Pereira et al. (2003:164) - el adiestramiento teórico-metodológico y la credencial constituyen el mayor incentivo para quienes tengan aspiración en un futuro de competir por un cargo académico.

En esencia, las condiciones laborales que tienen los auxiliares de investigación se encuentran respaldadas por el modelo burocrático clientelar que prevalece en la gestión universitaria, las cuales conllevan a la flexibilidad y precarización del trabajo que realiza el personal de apoyo.

\section{Elementos de flexibilidad laboral en el trabajo de los auxiliares de investigación}

Las relaciones de trabajo en la actualidad se encuentran influidas por las nuevas tecnologías utilizadas en los procesos productivos y la reestructuración organizativa, cuyo aspecto esencial se dirige a transferir algunas funciones de apoyo a trabajadores totalmente flexibilizados. En este contexto la flexibilidad laboral, según la Organización de Cooperación y Desarrollo Económico citado por Saravia (1998:2), adquiere relevancia como un conjunto de medios destinados a mejorar la eficiencia de las organizaciones y su capacidad de adaptación a las variaciones del medio donde se desenvuelven.

Las estrategias flexibilizadoras se reflejan en la reducción de la jornada laboral, contratación por tiempo determinado, ausencia de seguridad social, posibilidad de realización del trabajo fuera del espacio organizacional; en esencia, la flexibilización laboral se fundamenta en la ausencia de relaciones permanentes o de dependencia con el empleador.

Estas tendencias conducen, en la mayoría de los casos, a la precarización de un número significativo de trabajadores "periféricos" con respecto a trabajadores permanentes "núcleo" que llevan a cabo los procesos esenciales de la organización. Señala Sotelo (1996:124-125) que la precarización laboral es un concepto destinado a señalar los problemas que enfrentan los trabajadores, tanto calificados como no calificados, de empresas públicas o privadas, de los sectores formal e informal 
Flexibilidad laboral en el trabajo

investigativo: auxiliares de investigación

y que se traduce en bajos salarios, deterioro de la calidad del empleo, subempleo, prolongación desmedida de las jornadas de trabajo y, sobre todo, en deterioro de los derechos laborales.

A su vez, la flexibilidad laboral es un fenómeno social cuya definición tiene un carácter polisémico, dadas las múltiples connotaciones que adquiere el término en el marco de procedimientos, métodos, procesos, estrategias asociadas al trabajo. Dentro de la organización pública, como las universidades, las estrategias laborales flexibles se debaten en una constante contradicción entre elementos rígidos y flexibles.

La rigidez se manifiesta en excesiva definición de las tareas, estricto apego a la normativa y a las rutinas de trabajo, elementos propios del modelo burocrático clientelar imperante en las universidades públicas venezolanas; mientras que los elementos flexibilizadores introducen el énfasis en los resultados más que en los procesos. Ermida (1999: 139-140) detalla algunas de las características de una relación flexible de trabajo: segmentación de la mano de obra, individualización, descentralización del trabajo, inestabilidad en el empleo y abaratamiento del costo de trabajo.

Los elementos anteriormente señalados involucran cambios dentro de la organización pública, los cuales pueden ser generales o parciales. En este último aspecto se centra nuestra atención, al describir características flexibles y/o precarias de un cierto grupo de trabajadores en el ámbito universitario que se desempeñan en una función particular - la investigación - cuya dinámica propia también articula elementos flexibles en la organización del trabajo investigativo.

La organización del trabajo investigativo en la Universidad del Zulia se realiza a través de equipos de trabajo constituidos por personal académico y de apoyo a la investigación donde se llevan a cabo tareas intelectuales y operativas; estas últimas son realizadas, la mayor parte de las veces, por el personal de apoyo a la investigación. Cuando ellos tienen participación intelectual en la elaboración de ponencias y artículos, la división entre el trabajo intelectual y el operativo se diluye.

Por el contrario, cuando no se le proporciona al personal de apoyo participación en las actividades intelectuales, el producto que de ellas se genera le pertenece a los académicos: responsable del proyecto o programa, investigador principal o investi- 
gadores auxiliares. Ahora bien, el trabajo investigativo tiene características particulares que lo hacen atípico porque el usufructo generado - conocimiento científico- le pertenece a quien lo realiza, contrariamente a otro tipo de trabajo remunerado, en el cual el producto o servicio concebido le es ajeno al trabajador en la medida en que los beneficios de su labor pertenecen al dueño de los medios de producción.

Tal particularidad le imprime a la organización del trabajo investigativo una difusa subordinación del trabajo intelectual, pues todo integrante del equipo indistintamente de su ubicación jerárquica de referencia está llamado a contribuir a la creación de conocimientos tomando en cuenta que la ciencia como trabajo tiene de acuerdo con Vessuri (1995:21) carácter dinámico, colectivo y provisorio que posibilita la unión transitoria entre redes de trabajo, cuyos integrantes se reúnen para tratar un problema específico, una vez finalizado se disuelven y, posteriormente, formarán parte de otras redes.

Con base en lo anterior, la división tradicional del trabajo (intelectual/manual) en los equipos de investigación se desvanece y predomina entonces la actividad intelectual en búsqueda de conocimiento. Por lo tanto, cuando al personal de apoyo - parte integrante del equipo - se le confina a actividades operativas el equipo de investigación se dualiza y surge una frontera entre trabajadores intelectuales y operativos de forma similar al trabajo fabril, donde la prioridad es evitar que los trabajadores piensen y sólo se dediquen a la ejecución de tareas manuales.

Esta situación es experimentada por buena parte de los auxiliares de investigación, lo cual de acuerdo con el contenido de su trabajo, los coloca en condiciones desventajosas con tendencias precarias de trabajo. Sus funciones se limitan mayoritariamente a lo operativo (trámites administrativos, transcripción de entrevistas, búsqueda de materiales bibliográficos, fotocopiado, entre otras). Tales actividades son básicas para el desarrollo de todo proyecto de investigación; sin embargo, bajo la visión colectiva de la ciencia, es necesario que también participen en la creación de conocimientos para beneficio del proyecto y de su formación teórica-metodológica, lo cual permitiría dar continuidad a su crecimiento profesional en el campo de la investigación.

Existen otras condiciones que denotan rasgos de flexibilidad laboral que propician condiciones precarias de trabajo, a continuación se enumeran. 
Flexibilidad laboral en el trabajo

investigativo: auxiliares de investigación

\subsection{El carácter temporal de los proyectos y programas de investigación}

Los proyectos y programas de investigación financiados por el CONDES tienen una duración de un año y pueden ser prorrogados por seis meses, incluso pueden ser renovados por un año más. Los auxiliares de investigación pueden ser incorporados en cualquier momento de vigencia de los proyectos y programas y desincorporados a juicio del investigador responsable.

Se observó que durante los años 2003 y 2004, el 50\% de ese personal no sobrepasó el año de permanencia, tal como lo demostraron los informes finales de investigación. Esto se puede deber al escaso incentivo económico que existe para tales profesionales y también a la discrecionalidad que posee el investigador responsable con respecto al tiempo de contratación, el cual puede ser más corto con relación a la vigencia del proyecto y programa.

\subsection{La incertidumbre en la continuidad de la relación laboral}

Los auxiliares entrevistados afirmaron que es poca la certeza que tienen de continuar en una siguiente investigación; ello queda sujeto a la disponibilidad presupuestaria para nuevos proyectos o programas, así como a la decisión de los investigadores responsables de incluirlos en la renovación o apertura de nuevos proyectos o programas.

\subsection{La escasa remuneración}

La remuneración percibida no alcanza el salario mínimo. Reciben ciento setenta mil bolívares por una dedicación de cuarenta horas semanales. Algunos investigadores de trayectoria entrevistados expresaron que la baja remuneración dificulta la captación y la permanencia de auxiliares (en los proyectos o programas) profesionales talentosos que podrían ser formados como personal de investigación.

A partir de la gestión 2000-2004 se flexibiliza la remuneración del personal de apoyo a la investigación a través de la creación de la política a destajo. Mediante tal modalidad ese personal sería remunerado por las actividades por las cuales se le contrata y por un tiempo menor al de la duración de la investigación. De acuer- 
do con Bozo de Carmona (2004) - coordinadora-secretaria del CONDESesta política permite al investigador responsable remunerar al personal de forma más equitativa.

$\mathrm{Al}$ respecto, es decisión del investigador responsable acoger esta política de remuneración. La mayoría de los investigadores, en busca de una mayor permanencia de ese personal, opta por pagar montos que no sobrepasen la cantidad máxima mensual (Bs. 250.000) ${ }^{6}$ que le permite los fondos asignados por el CONDES para pago de personal. ${ }^{7}$ En consecuencia, la remuneración que reciben es precaria. ${ }^{8}$

Una muestra tomada de los proyectos aprobados, después de la aprobación de esa política, evidencia que el $76 \%$ de los investigadores responsables no se acoge al pago por destajo.

\subsection{La individualización en la relación de trabajo}

La normativa universitaria vigente no contempla regulaciones para la contratación de personal de apoyo. La relación de trabajo entre el auxiliar y el investigador responsable es de carácter individual y no implica beneficios socioeconómicos de ninguna índole.

En esencia, la condición de precariedad laboral de los auxiliares de investigación es el resultado de fricciones entre la flexibilidad propia de la actividad investigativa y la rigidez administrativa de un modelo universitario en el cual la actividad científica detenta un carácter marginal. En consecuencia, la regulación de la situación laboral de este personal se encuentra afectada por el desinterés de los actores decisores de la política científica universitaria y de investigadores.

${ }^{6}$ Esta cantidad representa 1,217 pesos mexicanos aproximadamente.

${ }^{7}$ La cantidad máxima que se asigna a la partida pago de personal es de Bs. 3.000.000,00 (14,609.00 pesos mexicanos) para cada año de vigencia del proyecto o programa, sin considerar el periodo de prórroga.

8 Esta aseveración se sustenta en lo siguiente: La remuneración máxima mensual no alcanza el salario mínimo (Bs. 405.000 equivalente a 1,987 pesos mexicanos). La asignación máxima anual del CONDES para el pago del personal es de Bs. 3.000.000, los cuales deben ser distribuidos entre el personal de apoyo (colaboradores y auxiliares de investigación); por tanto, la asignación máxima del auxiliar puede disminuir en función de la cantidad de personal de apoyo que tenga el proyecto o programa. 
Flexibilidad laboral en el trabajo

investigativo: auxiliares de investigación

\section{A modo de conclusión}

Los auxiliares de investigación conforman un grupo laboral precario por carecer de estabilidad, seguridad social y remuneración acorde con sus actividades y horas de trabajo. Más aún, luego de formados, no tienen certeza de llegar a ocupar un cargo académico debido a que la experiencia en docencia es uno de los requisitos más valorados en los concursos universitarios que, generalmente, son muy escasos.

En la gestión 2000-2004 se observaron ciertas medidas de flexibilidad como es la implementación de la jornada de trabajo a destajo en búsqueda de ventajas remunerativas. Sin embargo, esta medida reduce la permanencia del auxiliar en la investigación, adquiriendo un carácter utilitario que coarta la posibilidad de formación, desarrollo y maduración intelectual necesaria en la investigación.

Las condiciones de tipo financiero, organizativo y cultural de las universidades tales como la influencia de un modelo administrativo burocrático populista, la escasa atención que recibe la actividad de investigación, la poca incidencia de investigadores en las decisiones del consejo universitario y el papel del CONDES como ente financiador - mas no coordinador de la actividad investigativa - han limitado el diseño de políticas dirigidas al mejoramiento de la remuneración y condiciones de trabajo de los auxiliares de investigación.

En este contexto, el ethos $^{9}$ investigativo pierde arraigo en las nuevas generaciones en la medida que se les dificulta a profesionales la permanencia y dedicación a la actividad investigativa en proyectos y/o programas. De esta manera la investigación se torna una labor elitesca en la cual unos pocos están dispuestos a trabajar en condiciones desventajosas en comparación con otras áreas de la actividad profesional.

\footnotetext{
9 [...] un complejo emocionalmente teñido de reglas, prescripciones, costumbres, creencias, valores y presuposiciones que se consideran obligatorios para el científico.” (Merton, 1985).
} 


\section{Referencias bibliográficas}

BANCO CENTRAL DE VENEZUELA (1972, 1974,1978), Informes anuales. Venezuela.

BOZO DE CARMONA, Ana Julia (2004), Directora de la Secretaría del Condes 2000-2004, entrevista realizada el 7 de octubre de 2004.

CONSEJO DE DESARROLLO CIENTÍFICO Y HUMANÍSTICO (2004), Normas que rigen los distintos programas de apoyo a la investigación, Universidad del Zulia, Maracaibo, Venezuela.

CONSEJO UNIVERSITARIO (1998), Normas operativas para el cofinanciamiento de los becarios académicos, estudiantes de pregrado, asistentes (egresados de maestría), auxiliares de investigación (egresados de pregrado), Universidad del Zulia, Maracaibo, Venezuela.

, Reglamento de ingreso y concurso universitario, nota al pie.

, (1995), Normas de Operación del Consejo de Desarrollo Científico y Humanístico, Universidad del Zulia, Maracaibo, Venezuela.

CÓRDOVA JAIMES, Édgar (2002), “La flexibilización laboral en los entes descentralizados de competencias exclusivas", en Revista Venezolana de Ciencias Sociales, Universidad Nacional Experimental Rafael María Baralt (UNERMB), vol. 4, No.2, pp.129-145.

ERMIDA U., Oscar (1999), “Globalización y relaciones laborales”, Revista Venezolana de Gerencia, No. 9, Universidad del Zulia, Maracaibo, Venezuela.

FERRER Y ARROYO, Mercedes (2000), “Gestión y marketing del capital intelectual. Estrategia para posicionar los postgrados en el siglo XXI", en Revista Venezolana de Gerencia, año 5, No. 12, Ediciones Astro Data, Maracaibo, Venezuela. 
GAILLARD, Jacques (1994), "El comportamiento de los científicos y de las comunidades científicas", en Una búsqueda incierta. Ciencia, Tecnología y Desarrollo. Compiladores Jean-Jacques, Francisco Sagasti Salomón y Céline Sachs, Fondo de Cultura Económica, México.

JAUA MILANO, Elías (1997), "Del fordismo a la flexibilidad laboral: supuestos, crisis y realidades de la regulación social", en Revista Venezolana de Economía y Ciencias Sociales, vol.3, No.2-3, pp. 84-111.

LOMBARDI, Ángel (2004), Entrevista personal realizada el día 14 de marzo de 2004.

LUCENA, Héctor (2004), Relaciones del Trabajo en el Nuevo Siglo. Editorial Tropikos, Caracas, Venezuela.

MERTON, Robert K. (1985), La sociología de la ciencia, tomo 2, Alianza Universitaria, España.

MONTILLA, José Alberto y María Cristina Parra (1994), "Populismo y universidad: La marginalización de la investigación", Revista Estudios de Coyuntura, Universidad del Zulia, Maracaibo, Venezuela, pp. 65-73.

PEREIRA, Lilia Suárez, Wendolín Suárezy Elizabeth Boscán (2003), "Formación de estudiantes en investigación. Una experiencia del CEE," Revista Venezolana de Ciencias Sociales, año 1, No. 2, Universidad Nacional Experimental de Guayana, Ciudad Guayana, Venezuela.

ROMERO, Romer (1994), "Reflexiones sobre los problemas de la cultura científica: El caso de la Universidad del Zulia (Editorial)", Revista Acta Cientifica Venezolana, No. 45, pp. 1-3.

ROMERO, Maria Gracia (2004), Entrevista realizada el 1 de noviembre de 2004, Maracaibo, Venezuela.

SARABIA, Enrique (1998), "La situación laboral del personal de la administración pública CLAD", Documentos reuniones internacionales, No. 1, Caracas, Venezuela. 
SOTELO, Adrián (1996), "Globalización y precariedad del trabajo en México", Ediciones El Caballito, México.

VESSURI, Hebe (1995), La academia va al mercado. Relaciones de cientificos académicos con clientes externos, Fondo Editorial Fintec, Venezuela.

SUÁREZ PICÓN, Cira et al., (2001), "Relaciones de trabajo en el contexto de la globalización”, Revista Venezolana de Gerencia, año 6, No.15. đ 